\title{
Dancing the two-step with SAM
}

The outer membranes of mitochondria contain channel proteins with $\beta$-barrel structures. The precursors of $\beta$-barrel proteins are synthesized in the cytosol and are imported into mitochondria by the general translocase TOM and the sorting and assembly machinery (SAM) complex. But how $\beta$-barrel proteins are sorted and integrated into the outer membrane is poorly understood. Kutik et al. now propose a two-step mechanism whereby a signal sequence drives the association of the $\beta$-barrel protein with SAM, and the $\beta$-barrel protein is then integrated into the lipid phase of the outer membrane.

The authors screened for a potential sorting signal of $\beta$-barrel precursors by deleting $\mathrm{N}$ - and $\mathrm{C}$-terminal portions of the essential $\beta$-barrel protein Tom 40 and by monitoring the import of labelled constructs into isolated mitochondria. Tom 40 constructs with $\mathrm{C}$-terminal truncations were defective in associating with the SAM complex, and a conserved signal sequence, consisting of a large polar residue, an invariant Gly and two large hydrophobic residues, was identified in the most $\mathrm{C}$-terminal predicted transmembrane $\beta$-strand.

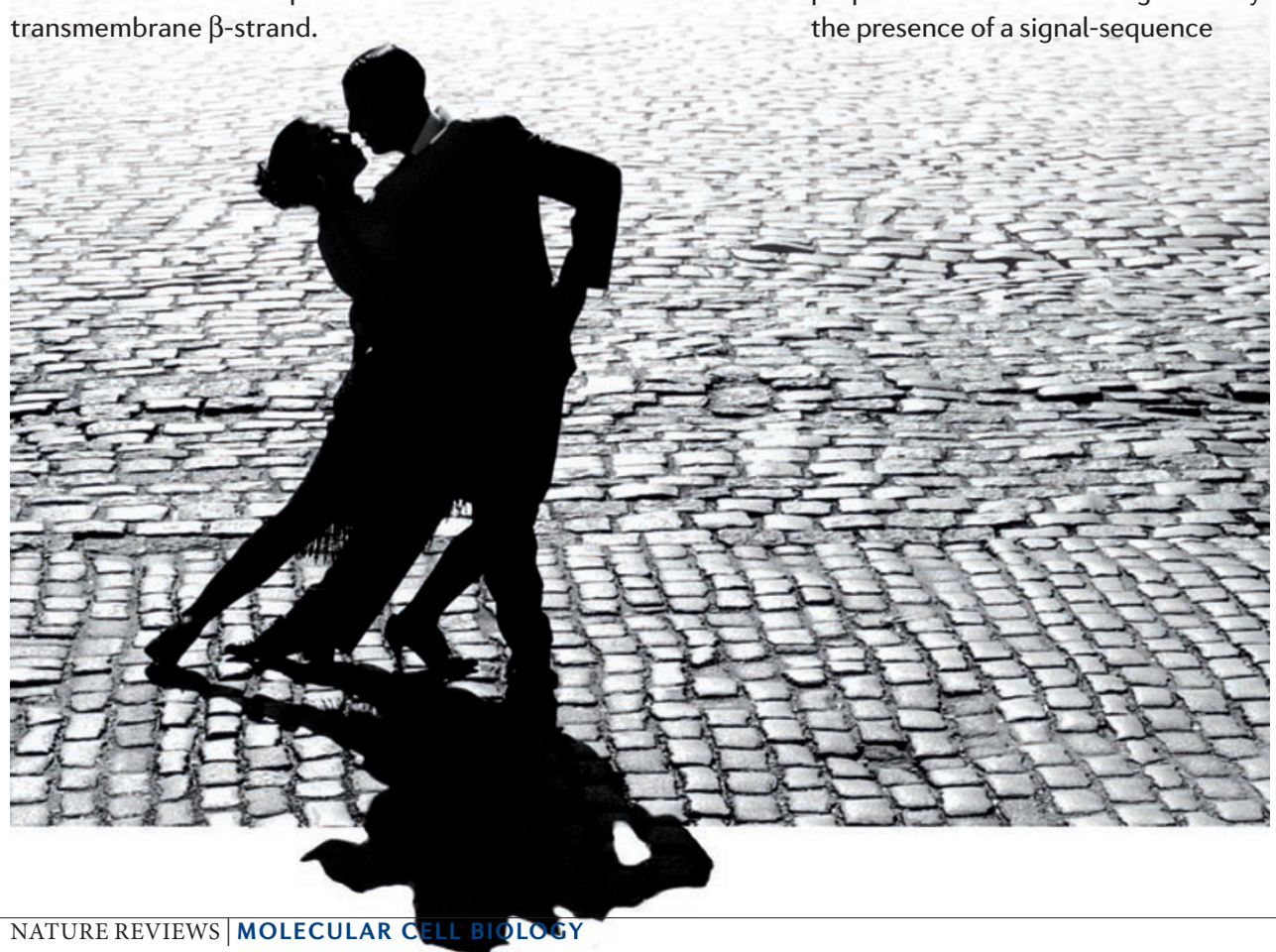

By mutating each of the four conserved signal-sequence residues, Kutik et al. established that the polar and hydrophobic residues were important for sorting Tom 40 to SAM. By contrast, mutating the invariant Gly residue did not affect the formation of the Tom 40 complex with SAM, but instead prevented membrane insertion. So, the four conserved residues of the signal sequence are involved in distinct functions that may be part of a two-step mechanism.

These findings were confirmed in other $\beta$-barrel proteins, demonstrating the generality of the signal sequence. This was further demonstrated by creating hybrids of $\beta$-barrel proteins by signal swapping and testing their functionality, and by competition experiments with a synthetic signalsequence peptide. A yeast strain that lacked endogenous Tom 40 was viable in the presence of plasmid-expressed wild-type Tom40, but not when a signal-sequence mutant of Tom40 was expressed. The signal sequence of Tom 40 is therefore essential for cell viability.

Reconstitution of the SAM complex into liposomes led to the formation of a channel, the gating properties of which were regulated by the presence of a signal-sequence peptide. By contrast, the activity of the channel that was formed by $\underline{\operatorname{Sam} 50}$ on its own remained unaffected by the signal-sequence peptide. The authors therefore postulated that the other essential SAM subunit, Sam35, may be required for recognition of the signal sequence.

However, this possibility creates a topological problem: Sam 35 is thought to be located on the cytosolic surface of the outer membrane, whereas $\beta$-barrel precursors are inserted into the outer membrane from the inter-membrane space. The authors concluded from various protection studies using proteases and amino-acid-modifying agents that Sam 35 is embedded into the outer membrane by its close association with the membrane domains of Sam50, and thus the $\beta$-barrel precursors can directly access Sam35. Moreover, binding of the SAM complex in isolated mitochondria by the signal-sequence peptide was blocked in Sam35-deficient mutant organelles, indicating that Sam35 is indeed required for signal-sequence recognition. This finding was confirmed in vitro with purified Sam35 and a signal-sequence peptide.

On the basis of these findings, the authors proposed that translocation of $\beta$-barrel precursors into SAM is initiated by binding of the most C-terminal $\beta$-strand - which contains the signal sequence - to Sam 35 . Signal binding leads to opening of the SAM channel and insertion of the $\beta$-barrel precursor into the channel. The precursor is then laterally released from the SAM complex into the outer membrane, and membrane insertion relies on conserved Gly residues in the signal sequence and on other $\beta$-barrel strands.

Arianne Heinrichs

ORIGINAL RESEARCH PAPER Kutik, S. et al. Dissecting membrane insertion of mitochondrial $\beta$-barrel proteins. Cell 132, 1011-1024 (2008) FURTHER READING Rehling, P. et al. Mitochondrial import and the twin-pore translocase. Nature Rev. Mol. Cell Biol. 5, 519-530 (2004) 